\title{
CULTURA Y ECONOMÍA EN ÉPOCAS DE GLOBALIZACIÓN
}

\author{
José Marsano Delgado
}

\section{RESUMEN}

Se expone la interrelación que existe entre la economía y la cultura dentro del contexto de un mundo globalizado. Se analiza también la necesidad de crear un instrumental pertinente, con la finalidad de que el intercambio cultural no sufra perjuicios propios del intercambio económico y de otras razones ajenas como el proteccionismo ideológico.

Palabras clave: cultura, OMC, GATT, Ronda de Uruguay, NAFTA, TLC, economías a escala, industrias culturales, diversidad cultural.

\section{INTRODUCCIÓN}

La preservación de la diversidad cultural dentro de un contexto de globalización de la economía ha cobrado gran importancia en tiempos recientes, hecho que hubiese sido difícil de pensar, o mejor dicho de imaginar, pocos años atrás. El caso es que esta problemática ya genera preocupaciones, al igual que los fenómenos climatológicos, económicos y otros tantos como el de las buenas prácticas comerciales. Como señalábamos, esta inquietud es relativamente nueva si la comparamos con los pensamientos de la liberalización del comercio a través de los acuerdos comerciales multilaterales o regionales, que ya datan de más de sesenta años, y en cuyo marco se han producido numerosas referencias a la cultura, aunque entendida aparentemente en forma ambigua y poco recíproca. "La ambigüedad que todavía rodea a su condición" (Berner y Sauvé, 1999).

La inclusión de la cultura en este tipo de convenios se debe a los progresos en la liberalización del comercio, gracias a la mayor institucionalización de la Organización Mundial del Comercio (OMC) durante la ronda de Uruguay, que dio origen a la más amplia reforma del sistema mundial de comercio desde la creación del Acuerdo General sobre Aranceles Aduaneros y Comercio (GATT, por sus siglas en inglés) a finales de la Segunda Guerra Mundial. A pesar de sus dificultosos avances, la Ronda de Uruguay produjo desde el inicio algunos resultados plausibles. En solo dos años los participantes se pusieron de acuerdo sobre un conjunto de reducciones de los derechos de importación a los productos tropicales (que son exportados sobre todo por países en desarrollo). También revisaron las normas para la solución de diferencias, y algunas medidas fueron aplicadas de inmediato.

Establecieron además la presentación de informes periódicos sobre las políticas comerciales de los miembros del GATT (convenio multilateral creado en la Conferencia de La Habana en 1947 y firmado en 1948, ante la necesidad de establecer 
un conjunto de normas comerciales y concesiones arancelarias, y considerado como el precursor de la OMC. El GATT era parte del plan de regulación de la economía mundial tras la Segunda Guerra Mundial e incluía la reducción de aranceles y otras barreras al comercio internacional), medida que se consideró importante para lograr que los regímenes comerciales fueran transparentes en todo el mundo. Entre sus competencias se incluía colaborar en las soluciones a la prestación de servicios y en las inversiones, incluso en materia de productos culturales.

Como señala François Rouet (2000), "la globalización atañe cada vez más a la cultura", en función a la mayor circulación de productos culturales a escala mundial, y al desarrollo -en la cultura y en la comunicación- de empresas de gran tamaño que encarnan esta globalización; y, por otro lado, en función de aspiraciones reforzadas de que se mantengan y se fomenten expresiones regionales, nacionales o locales. Ante todo ello cabe hacerse la siguiente pregunta: ¿Puede y debe la cultura insertarse en el contexto de la liberalización del comercio y de la inversión, al igual que los demás bienes y servicios producidos mundialmente?

El hecho de que la cultura cuestione la lógica del comercio mundial conduce a preguntarse a qué campo podría pertenecer. Las particularidades que reivindica, incluyendo los objetivos de las políticas sectoriales que se realizan bajo la denominación de "políticas culturales", nos plantean grandes interrogantes: ¿Qué es la cultura? ¿Cuál es su papel en la sociedad? ¿Las evoluciones económicas en curso amenazan la diversidad cultural? ¿Qué formas de cooperación internacional exige la promoción de la diversidad cultural?

Testimonios de la creciente importancia que adquieren las anteriores acotaciones los podemos encontrar en los discursos políticos. Así, por ejemplo, en el comunicado final de la reunión del G-8 (grupo de países industrializados del mundo cuyo peso político, económico y militar es muy relevante a escala global, conformado por Alemania, Canadá, Estados Unidos, Francia, Italia, Japón, Reino Unido y Rusia) en Okinawa, en julio del año 2000, se pueden leer no menos de cuatro párrafos concernientes a la diversidad cultural, declarando, entre otras cosas, que:
[...] la diversidad cultural es una fuente de dinamismo social y económico que puede enriquecer la vida humana en el siglo XXI suscitando la creatividad y fomentando la innovación. Reconocemos y respetamos la importancia de la diversidad en la expresión lingüística y creadora. [...] La creciente interacción entre los pueblos, los grupos y los individuos aporta una mayor comprensión y una mayor apreciación de lo que es bueno e interesante en cada cultura. La promoción de la diversidad cultural refuerza el respeto mutuo, la inclusión y la no discriminación y lucha contra el racismo y la xenofobia. [...] Las tecnologías de la información ofrecen a las personas unas perspectivas sin precedentes para crear y compartir el contenido cultural y las ideas, a escala mundial y a bajo costo. La experiencia demuestra que la diversidad puede despertar la curiosidad, engendrar la iniciativa y aportar una contribución útil en las comunidades que buscan mejorar sus economías, en especial, mediante la utilización de los medios extraordinarios que la sociedad de la información pone a su disposición. [...] Para maximizar las ventajas de la interacción cultural, debemos alentar a nuestros pueblos a aprender a vivir juntos, favoreciendo el interés, la comprensión y la aceptación de las culturas diferentes.

\section{INTERACCIÓN ENTRE ECONOMÍAY CULTURA}

Uno de los problemas fundamentales que plantea la interacción entre la economía y la cultura se refiere al tratamiento y asignación de los productos culturales en los acuerdos comerciales internacionales. Por ahora el lugar asignado a tales productos en los acuerdos existentes se caracteriza por una evidente ambivalencia. Tratados en principio como cualquier otro producto, con bastante frecuencia -dependiendo de los contextos y materias- disfrutan de cláusulas derogatorias o de reservas. No obstante, los productos culturales son objeto de un tratamiento especial en cierto número de acuerdos multilaterales vinculados a la OMC: el GATT de 1994 para el comercio de mercancías, el AGCS para el comercio de servicios y el ADPIC para los derechos de propiedad intelectual relacionados con el comercio. Asimismo, algunos acuerdos de 
integración o comerciales, como el de la Unión Europea, el NAFTA, el TLC entre Estados Unidos y Perú, así como otros diversos acuerdos bilaterales, se han interesado por esta interrelación, buscando circunscribir el alcance de disciplinas comerciales en el sector, promover la producción cultural local o incluso favorecer diversas formas de cooperación en el ámbito cultural.

Dos visiones radicalmente opuestas sobre los productos culturales son objeto de este artículo. Una de ellas los consideraría como productos de entretenimiento similares, desde un punto de vista comercial, a cualquier otro y, por tanto, totalmente subordinados a las reglas del comercio internacional. La otra propondría que los productos culturales son bienes que transmiten valores, ideas y conocimiento; es decir, son instrumentos de comunicación social que contribuyen a forjar la identidad cultural de una colectividad y, por ello, deberían quedar excluidos del ámbito de los acuerdos comerciales internacionales. En definitiva pensamos que ninguna de estas dos posiciones es aceptable en un mundo globalizado.

\section{LOS PRODUCTOS CULTURALES COMO OBJETOS COMERCIALES}

Según Pierre Sauvé, experto en Derecho del Comercio Internacional:

Como objetos comerciales, los productos culturales difícilmente pueden quedar totalmente excluidos del alcance de los acuerdos comerciales. En efecto, desde el momento en que son explotados con el fin de obtener un beneficio comercial y que por lo tanto son objeto de intercambios en el plano internacional, deberá de inferirse que entran en juego una serie de intereses $y$, en ocasiones, opuestos que solo pueden ser reconciliados dentro de un marco jurídico apropiado. Entre estas razones tendríamos que los principales países exportadores de productos culturales se opondrían a que estos últimos quedaran excluidos del marco jurídico que regula el comercio internacional. A la cabeza de este grupo encontramos en primer lugar a Estados Unidos, para quien el conjunto de bienes y servicios culturales supone un importante rubro de exportación, así como otros países como Brasil, México y Japón. Estos últimos seguramente no verían con buenos ojos que sus exportaciones pudieran ser objeto de marginación por motivos culturales y escapasen a todo control. Varios países que han desarrollado una presencia significativa en el mercado internacional de productos culturales, incluidas naciones como Canadá, España o Francia que, por otro lado, luchan para que se reconozca el carácter diferente de las industrias culturales en el comercio internacional, también podrían mostrarse inquietos ante un desarrollo de este tipo. Incluso a los países en vías de desarrollo, cuya producción cultural circula cada vez más en el mundo, les interesaría que se mantuviese una gran apertura mercantil en el ámbito cultural'.

Por otro lado, los países cuyo mercado interno no puede sostener una producción cultural diversificada y que necesitan productos culturales extranjeros para responder a su demanda interna, también se benefician de tener asegurado el acceso sin discriminación a la producción cultural extranjera. Pero este acceso no les sería garantizado si los productos culturales tuvieran que ser totalmente excluidos del marco jurídico que regula el comercio internacional. Incluso un principio de no discriminación tan fundamental como el del tratamiento de nación más favorecida no podría ser invocado en tales circunstancias.

Más allá de estas consideraciones exclusivamente económicas, también es importante señalar que el hecho de eximir completamente a los productos culturales de los acuerdos comerciales internacionales abre peligrosamente la puerta, en el plano jurídico, a restricciones más justificadas por razones de proteccionismo comercial o incluso ideológico.

\footnotetext{
1 "Cultura y economía: los retos en juego. Tres espacios lingüísticos". Organización de Estados Iberoamericanos, http://www.campus-oei. org/tres_espacios/icoloquiol0.htm, 22.2.2012.
} 
Pero si bien una eliminación total de los productos culturales del contexto del comercio internacional no parece una solución lógica, esto tampoco implica que haya que adoptar una visión exclusivamente comercial de los productos culturales. Al contrario, esta visión sería arriesgada. En efecto, desde hace varios años se puede ver que la producción cultural se está convirtiendo cada vez más en un asunto propio de las industrias culturales. Sin embargo, este fenómeno de industrialización y de comercialización de la producción cultural, si bien ha ampliado considerablemente el acceso a la producción cultural y ha permitido el desarrollo de nuevos soportes de difusión, también puede tender, paradójicamente, a limitar la oferta de productos culturales, debido a la masificación de las industrias culturales, en la que por razones estrictamente económicas aquellas manifestaciones culturales que la industria no considere rentables desde el punto de vista comercial podrían quedar fuera del mercado, en perjuicio de la diversidad cultural.

\section{UNA PROPUESTA DE SOLUCIÓN}

Una solución ideal, que a largo plazo puede aportar una respuesta al conflicto actual sobre el lugar que deben ocupar los productos culturales en los acuerdos comerciales internacionales, sería un convenio especial sobre los intercambios internacionales en el sector cultural. Tal convenio articularía de forma clara la justificación y los límites de un tratamiento especial para los productos culturales insistiendo en la necesidad de preservar la diversidad cultural.

¿Cuáles podrían ser los objetivos de este instrumento? Según Sauvé:

[...] en el plano de los esfuerzos diplomáticos, el principal desafío consiste en formar una alianza de países que compartan preocupaciones similares en materia de política cultural para influir en las decisiones que deben ser tomadas en diversos espacios multilaterales, regionales $\mathrm{o}$ incluso bilaterales y promover un enfoque de las relaciones internacionales que favorezca la diversidad cultural.

En el plano cultural propiamente dicho, el objetivo debería ser lograr que se reconozca la importancia de la diversidad cultural y que se acepte la necesidad de un tratamiento diferenciado de los bienes y servicios culturales en los acuerdos comerciales internacionales. A estos objetivos generales se añadiría de forma más explícita una referencia a la circulación, lo más libre posible, de los productos culturales².

En efecto, habría que evitar que la diversidad cultural deje de correr el riesgo de una exclusión y medidas de restricción de los intercambios o de las inversiones en el campo cultural. Si ese fuera el caso, esta iniciativa correría el riesgo de quedar rápidamente relegada al olvido. En efecto, como bien señala Sauvé: "La diversidad cultural no se puede concebir sin apertura a los productos, ideas, valores, métodos de producción y redes de distribución de las demás culturas, ya que estas alimentan y enriquecen las culturas nacionales" ${ }^{\prime 3}$.

\section{CONCLUSIONES}

- Como se ha podido ver, la cultura mantiene, por naturaleza, una relación ambigua con la economía, el comercio, la inversión, la competencia y sus modos y reglas de funcionamiento. Por un lado la cultura como conjunto de actividades pertenece, a todas luces, al ámbito de la economía en el sentido de que todas las actividades calificadas como culturales -incluso las menos mercantiles y subvencionadas por los poderes públicos- pertenecen a la economía, conocen la articulación entre oferta y demanda.

- La consideración de la interrelación entre el comercio y la cultura debe superar el ámbito por naturaleza restringido de la política comercial, que se debe al hecho de que, tal y como ya se señaló, el juego normal de las fuerzas del mercado no conduce necesariamente a un ideal social en materia de diversidad cultural. En efecto, las deficiencias del mercado ocasionadas por la combinación de economías a escala no llevan 
forzosamente al mantenimiento de la diversidad de las culturas y de las producciones culturales.

- Los estados deben verse en la necesidad de que en el orden político se haga valer la superioridad de la interrelación entre comercio y cultura sobre el orden comercial y económico, la debe ser reconocida por la comunidad internacional. La razón obedece, como ya lo hemos señalado a lo largo del artículo, a la necesidad de defender y proteger la diversidad cultural.

- En el área del comercio internacional se debe reconocer el carácter dual de los productos culturales, los que al mismo tiempo son objetos comerciales e instrumentos de comunicación social. El desarrollo de instrumentos flexibles es necesario para evitar que el intercambio cultural en la comunidad internacional se vea perjudicado por razones económicas o ideológicas.

\section{Bibliografía}

Balio, Tino

1995 Grand Design: Hollywood as a Modern Business Entreprise, 1930-1939. Los Angeles / Berkeley: California University Press.

Ballart, Joseph

2001 Gestión del patrimonio cultural. Barcelona: Ariel.
Berner, Ivan y Pierre Sauvé 1999 "Le cycle de négociation du millénaire: quels enjeux pour la francophonie?". Documento de trabajo para la concertación francófona en el marco de las negociaciones de la OMC de la ronda de Seattle. Agencia intergubernamental de la francofonía, octubre; en especial, parte II, pp. 5 y ss.

Colbert, François y Manuel Cuadrado 2003 Marketing de las artes y la cultura. Madrid: Ariel Patrimonio.

Ewen, Stuart

1977 Captains of Consciousness. Advertising and Social Roots of the Consummer Culture. New York: McGraw-Hill.

García Canclini, Néstor

1999 La globalización imaginada. Buenos Aires: Paidós.

McLuhan, Marshall y B. R. Powers

1989 The Global Village. Transformations in World, Life and Media in the $21^{\text {st }}$ Century. New York: Oxford University Press.

Rouet, François

2000 "Le soutien aux industries culturelles dans I'aire francophone: Modalités, Enjeux et Incidences". Texto elaborado para la II concertación intergubernamental de la francofonía sobre el tema: La promoción de la diversidad cultural. París (12 de diciembre). UNESCO, Commerce, culture et mondialisation: questions et réponses, abril. 